\title{
Hydration Properties and Solvent Effects for All-Atom Solutes in \\ Polarizable Coarse-Grained Water
}

\section{Supporting Information}

Xin Cindy Yan, Julian Tirado-Rives, and William L. Jorgensen*

Department of Chemistry, Yale University, New Haven, Connecticut 06520-8107

Table S1. Computed Properties of Polarizable Martini CG water at $27^{\circ} \mathrm{C}$ and $1 \mathrm{~atm}$, using MC and MD simulations. ${ }^{a}$

\begin{tabular}{lllll}
\hline method & $\mathbf{M C}^{b}$ & $\mathbf{M D}^{c}$ & ref. $^{d}$ & exptl. $^{e}$ \\
\hline \# of particles & 1998 & 1999 & & - \\
density & $1.049 \pm 0.000$ & $1.043 \pm 0.004$ & 1.043 & 0.997 \\
$\Delta \mathbf{H}_{\text {vap }}$ & $2.39 \pm 0.00$ & $2.37 \pm 0.01$ & - & 10.51 \\
$\boldsymbol{\kappa}$ & $8.80 \pm 0.53$ & 7.62 & - & 4.58 \\
$\boldsymbol{\alpha}$ & $10.76 \pm 0.76$ & 5.42 & - & 2.57 \\
$\mathbf{D}$ & - & $2.35 \pm 0.08^{f}$ & 2.5 & 2.27 \\
$\boldsymbol{\mu}$ & $5.09 \pm 0.03$ & $4.86 \pm 0.93$ & 4.9 & - \\
\hline
\end{tabular}

\footnotetext{
${ }^{a}$ Density in $\mathrm{g} \mathrm{cm}^{-3}, \Delta H_{\text {vap }}$ in $\mathrm{kcal} \mathrm{mol}{ }^{-1}, \kappa$ in $10^{-5} \mathrm{~atm}^{-1}, \alpha$ in $10^{-4} \mathrm{~K}^{-1}$, and $\mu$ in Debye. ${ }^{b} \mathrm{MC}$ simulations were performed with $70 \mathrm{M} / 200 \mathrm{M}$ configurations using the BOSS program. ${ }^{25 c} \mathrm{MD}$ simulations were performed following the standard protocol ${ }^{26}$ using the GROMACS program, version 4.5.4. ${ }^{d}$ Ref. $26 .{ }^{e}$ The experimental data were obtained at $298 \mathrm{~K}$ and $1 \mathrm{~atm}$. ${ }^{f}$ The selfdiffusion coefficient of individual water molecules was determined by dividing the diffusion coefficient of polarizable water bead by $4 .{ }^{26}$
} 

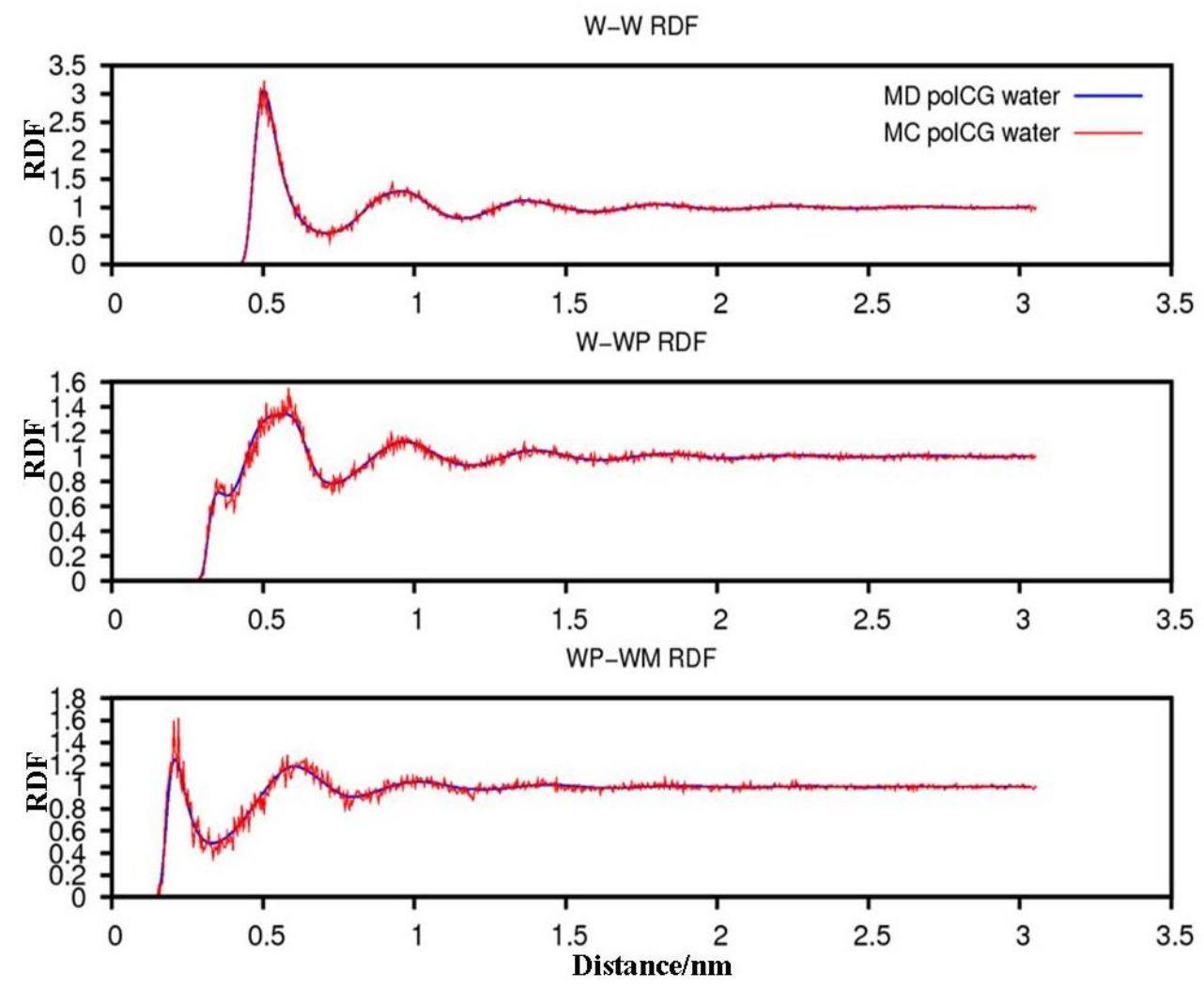

Figure S1. Plots of the radial distribution function for the polarizable Martini CG water in MC and MD simulations. W is the central particle of the CG water, and WP and WM are the particles bound to the central particle carrying positive and negative charges, respectively. 